\title{
Protección anticorrosiva de un convertidor de óxido natural (Mimosa tenuiflora) aplicado sobre productos de corrosión de un acero AISI 1018 Anticorrosive protection of natural rust converter (Mimosa tenuiflora) applied on corrosion products of AISI 1018 steel
}

\author{
David Enrique Arceo-Gómez ${ }^{1}$ (D) 0000-0001-7561-526X \\ Javier Reyes-Trujeque 2 (D) 0000-0002-8129-5343 \\ Ricardo Galván-Martínez ${ }^{3}$ (i) 0000-0003-2889-1135 \\ Ricardo Orozco-Cruz ${ }^{3}$ (D) 0000-0002-1983-2806 \\ ${ }^{1}$ Universidad Veracruzana, Centro de Investigación en Micro y Nanotecnología (MICRONA) \\ davarceo@gmail.com \\ ${ }^{2}$ Universidad Autónoma de Campeche, Laboratorio Nacional de Ciencias para la Investigación y \\ Conservación del Patrimonio Cultural, Centro de Investigación en Corrosión (LANCIC-CICORR) \\ ${ }^{3}$ Universidad Veracruzana, Instituto de Ingeniería \\ (c) Universidad De La Salle Bajío (México)
}

Palabras clave: corrosión; óxido; óxido natural; recubrimientos; aceros; protección; anticorrosión; convertidor; Mimosa tenuiflora; acero 1018; convertidores; patrimonio cultural metálico

Keywords: corrosion; rust; natural rust; coating; steel; protection; anticorrosion; converter; Mimosa tenuiflora; 1018 steel; converters; metallic cultural heritage

Recepción: 10 - 04 - 2021 / Aceptación: 02 - 06 - 2021

\section{Resumen}

Introducción: Un recubrimiento primario es aquel que es aplicado sobre la superficie de los aceros, como una primera capa de recubrimiento que permite mejorar la adherencia y el sellado de los sistemas protectores. Sin embargo, algunos de sus componentes son tóxicos para el ambiente y dañinos para la salud. Una alternativa amigable con el ambiente es el empleo de convertidores de óxido, obtenidos a partir de extractos naturales de plantas. Su uso puede llegar a tener un fuerte impacto en el ámbito industrial y resultan prometedores en la conservación del patrimonio cultural metálico. En este trabajo, se evaluó el efecto anticorrosivo de un convertidor de óxido obtenido a partir de un extracto de la planta Mimosa tenuiflora, aplicado sobre productos de corrosión de un acero 1018. La caracterización morfológica del convertidor de óxido se llevó a cabo por Microscopía Electrónica de Barrido (MEB). Por su parte, las propiedades anticorrosivas se evaluaron por Espectroscopía de Impedancia Electroquímica (EIE).

Método: Se utilizaron sustratos de acero AISI 1018 y se le aplicaron dos tratamientos de limpieza superficial: decapado químico y chorro de arena. Posteriormente, para formar productos de 
corrosión, las probetas se expusieron 90 días a la atmósfera urbano-marina de la ciudad de Boca del Río, Veracruz. El extracto utilizado como convertidor de óxido se obtuvo de la planta Mimosa tenuiflora y fueron preparadas dos formulaciones que se aplicaron por aspersión sobre la capa de productos de corrosión. Los productos de corrosión fueron caracterizados por Espectroscopía Raman, Difracción de Rayos X (DRX) y MEB-EDX. Por su parte, la morfología de la película convertida fue caracterizada por MEB. Las propiedades anticorrosivas de los productos de corrosión y el convertidor de óxido fueron evaluadas por EIE durante 24 horas de exposición en una solución de $\mathrm{NaCl}$ al $3.5 \%$ en peso.

Resultados: La capa de productos de corrosión está formada principalmente por goethita, lepidocrocita y hematita. Mediante MEB se observaron cambios en las características morfológicas de los productos de corrosión, los cuales cambian su aspecto superficial rugoso y poroso al de una superficie compacta y craquelada por acción del convertidor de óxido. Los resultados por EIE, mostraron que el tratamiento por chorro de arena sobre las probetas permitió una mejor adherencia de los productos de corrosión y convertidor de óxido, propiciando un aumento en la capacidad protectora en comparación con el tratamiento superficial por decapado químico.

Discusión o Conclusión: El convertidor de óxido natural mejora las propiedades protectoras de la capa de productos de corrosión. Aunado a lo anterior, una preparación superficial del sustrato con chorro de arena permitió mejorar la adherencia del convertidor de óxido. Este convertidor de óxido puede ser utilizado en el ámbito industrial y el patrimonio cultural metálico.

\begin{abstract}
Introduction: A primary coating is one that is applied on the surface of the steels as a first coating layer that allows better adherence and sealing of the protective systems. However, some of its components are toxic for the environment and harmful to the health of those who use them. An alternative that has been explored in recent years, which can be environmentally friendly, is the use of oxide converters obtained from natural plant extracts. Their use can have a strong impact in the industrial field and they show promise in the conservation of metallic cultural heritage. In this work, the anticorrosive effect of an rust converter obtained from an extract of the Mimosa tenuiflora plant applied on corrosion products of a 1018 steel was evaluated. The morphological characterization of the rust converter was carried out by Scanning Electronic Microscopy (SEM).
\end{abstract}


On the other hand, the anticorrosive properties were evaluated by Electrochemical Impedance Spectroscopy (EIE).

Method: AISI 1018 steel substrates were used and two surface cleaning treatments were applied: chemical pickling and sandblasting. Subsequently, to form corrosion products, the coupons were exposed for 90 days to the urban-marine atmosphere of the city of Boca del Río, Veracruz. The extract used as a rust converter was obtained from the Mimosa tenuiflora plant and two formulations were prepared that were applied by spraying on the layer of corrosion products. The corrosion products were characterized by Raman Spectroscopy, X-Ray Diffraction (XRD) and SEM-EDX. For its part, the morphology of the converted film was characterized by SEM. The anticorrosive properties of the corrosion products and the oxide converter were evaluated by EIS during 24 hours of exposure in a $3.5 \% \mathrm{NaCl}$ solution.

Results: The corrosion product layer consists mainly of goethite, lepidocrocite and hematite. By SEM, changes in the morphological characteristics of the corrosion products were observed, which change their rough and porous surface appearance to that of a compact and cracked surface due to the action of the rust converter. The results by EIE showed that the sandblasting treatment on the specimens allowed a better adherence of the corrosion products and rust converter, favoring an increase in the protective capacity compared to the surface treatment by chemical pickling.

Discussion or Conclusion: Natural rust converter improves the protective properties of the corrosion product layer. In addition to the above, a surface preparation of the substrate with sandblasting allowed better adhesion of the rust converter. This rust converter can be used in industrial environment and metallic cultural heritage.

\section{Introducción}

El acero al carbono se utiliza en grandes tonelajes para aplicaciones marinas, procesamiento químico, producción de petróleo, procesos de refinación, construcción y equipos de procesamiento de metales (Xu et al., 2019) que operan al aire libre, lo que genera condiciones que propician su corrosión. La corrosión es un fenómeno que a menudo se puede observar en grandes edificios o estructuras industriales y de transporte. Por definición, la corrosión es aquella interacción fisicoquímica entre un metal y su entorno que ocurre de manera espontánea, debido que todos los metales utilizados para construcciones técnicas tienen tendencia a perder electrones (es decir, a 
oxidarse) y a volver a su estado natural (termodinámicamente estable) formando óxidos, sales e hidróxidos (Jaramillo et al., 2019). La velocidad de corrosión del acero en dichos sistemas oscila entre 0.1 y $1 \mathrm{~mm} /$ año y son inaceptables desde un punto de vista técnico, ya que las estructuras rara vez se diseñan para una vida útil inferior a 50 años (Bozovic et al., 2020).

La corrosión atmosférica de las estructuras de acero al carbono origina la formación de una capa de óxidos y oxihidróxidos sobre la superficie metálica. Sus dos componentes principales de la capa de óxido son la lepidocrocita $(\gamma-\mathrm{FeOOH})$ y la goethita $(\alpha-\mathrm{FeOOH})$. Si hay ausencia de oxígeno debajo de esta capa, su concentración es limitada, se forma un óxido oscuro compuesto de magnetita $\left(\mathrm{Fe}_{3} \mathrm{O}_{4}\right)$, la cual no suele ser protectora, ya que en su presencia la corrosión del acero puede acelerarse mediante un efecto de acoplamiento galvánico entre el sustrato de acero y el óxido. En realidad, la formación de una capa de óxido compacta, altamente adhesiva y por lo tanto protectora sobre la estructura de acero no es común (Zhao et al., 2014), y pueden dificultar la aplicación de pinturas y por tanto, su rendimiento, pues genera una mala adherencia al sustrato metálico (Byrne et al., 2019). La protección y prevención contra la corrosión son muy importantes, ya que una parte considerable del producto nacional bruto de las naciones se invierte anualmente en acciones para evitar la corrosión. Entre los métodos de protección que son utilizados con mayor frecuencia se tienen los recubrimientos orgánicos e inorgánicos, la protección electroquímica y la inhibición de la corrosión (Li et al., 2018). En el caso de los recubrimientos, debe haber una buena preparación superficial del sustrato metálico para facilitar su anclaje; esto es esencial para proporcionar una mejor protección a largo plazo del sistema de recubrimiento. La preparación de la superficie implica la remoción total de óxido para alcanzar la etapa de metal blanco. Sin embargo, no siempre es posible conseguirlo, por inaccesibilidad de las estructuras o por consideraciones económicas (Collazo et al., 2008). El rendimiento de un recubrimiento orgánico depende principalmente del estado de la superficie metálica, así como de la agresividad del medio ambiente. La aplicación de una pintura a una superficie contaminada u oxidada da como resultado una falla temprana del recubrimiento (Vetere y Romagnoli, 1998). Entre las técnicas de limpieza de superficies, el chorreado abrasivo ofrece el mejor nivel de eliminación de óxido y aumenta la rugosidad superficial del sustrato, aumentando la adherencia de la película de recubrimiento (Matamala et al., 1993; Ocampo et al., 2004). En la industria automotriz y manufacturera, en la que generalmente se aplica pintura al acero, se usa una capa de fondo o primer para aumentar la adherencia y la calidad anticorrosiva de las pinturas (Matamala et al., 1993). A pesar de lo anterior, 
muchas de las pinturas primarias que protegen al acero de la corrosión, contienen pigmentos inhibidores como óxido de plomo o cromatos que tienen buenas propiedades anticorrosivas, pero debido a su alta toxicidad y potencial de causar daños ambientales, deben ser sustituidos por inhibidores ambientalmente más compatibles (Li et al., 2018; Feng et al., 2020).

Por otra parte, la estabilización de la capa de óxido antes de aplicar el revestimiento de pintura es una alternativa atractiva a la limpieza del acero, y en la actualidad es una tendencia mundial en la tecnología anticorrosiva.

Con el paso del tiempo, ha aparecido una amplia gama de convertidores de óxidos de hierro. La mayoría de estos productos se basan en ácidos fosfórico, tánico y gálico. Sin embargo, los resultados obtenidos con dichos productos comerciales son controvertidos (Feliu et al., 1993). Los convertidores de óxido son sustancias que se aplican a superficies corroídas provocando su pasivación, es decir, reaccionan con los óxidos de hierro que no pueden eliminarse por completo de la superficie metálica, convirtiéndola en una capa protectora adherente y factible para la aplicación de un recubrimiento (Barrero et al., 2001; Ocampo et al., 2004; Zhao et al., 2014; Saji, 2019). Se sabe que los agentes de conversión de óxido pueden ofrecer una mayor capacidad de prevención de la corrosión de superficies oxidadas a bajo costo, lo que evita la necesidad de emplear técnicas extensivas de limpieza de superficies, como el pulido con chorro de arena, y mejora la adhesión de la capa superficial. Su éxito depende de la naturaleza de las propiedades, tanto de la capa de óxido (óxido adherente total, espesor de la película de óxido, estructura y composición del óxido), como del convertidor de óxido (estructura, concentración, solubilidad, pH, capacidad de quelato con iones férricos) (Saji, 2019).

Entre los convertidores de óxido más estudiados por la comunidad científica se tienen los taninos, que son compuestos orgánicos naturales, no tóxicos y biodegradables que, se sabe, tienen la capacidad para inhibir el proceso de corrosión en muchos entornos, como en medios ácidos (Xu et al., 2019). De acuerdo con estos estudios, los taninos pueden ser utilizados como inhibidor y pretratamiento para recubrimientos anticorrosivos (Favre y Landolt, 1993; Ross y Francis, 1998; Favre et al., 1998; Barrero et al., 2001; Ocampo et al, 2004; Collazo et al., 2008; Ostovari et al., 2009; Silveira et al., 2012; Zhao et al., 2014; Li et al., 2018; Morteo et al., 2018; Xu et al., 2019; Jaramillo et al., 2019; Arceo et al., 2019; Bozovic et al., 2020). Los taninos son compuestos polifenólicos de origen vegetal y su estabilidad como convertidor de óxido depende de la 
temperatura, el disolvente, el tamaño de las partículas y de los procedimientos de extracción, que resultan específicos para diferentes especies de plantas (Byrne et al., 2019).

En este trabajo, se realizaron dos tratamientos superficiales a un acero 1018. El primero fue un decapado químico; y el segundo, un desbaste por chorro de arena o sandblast. Posteriormente, se hicieron crecer productos de corrosión atmosférica sobre las probetas de acero durante 90 días. Finalmente, se les aplicó la formulación del convertidor de óxido sobre los productos de corrosión. El objetivo fue evaluar las propiedades anticorrosivas de un convertidor de óxido obtenido del extracto de la planta Mimosa tenuiflora, aplicado sobre productos de corrosión de un acero 1018. La caracterización superficial de la película convertida y de los productos de corrosión se realizó mediante Microscopía Electrónica de Barrido acoplada a emisión de Energía Dispersiva de Rayos $\mathrm{X}$ (MEB-EDX). Las fases cristalinas de los productos de corrosión se identificaron mediante Espectroscopía Raman y Difracción de Rayos X (DRX); y las propiedades anticorrosivas fueron evaluadas por la técnica de Espectroscopía de Impedancia Electroquímica (EIE).

\section{$\underline{\text { Método }}$}

\section{Preparación de las muestras}

Los electrodos de trabajo utilizados fueron probetas de acero AISI 1018 con dimensiones de $3.5 \mathrm{x}$ $5 \mathrm{~cm}$, las cuales se prepararon superficialmente por decapado químico, utilizando como referencia la norma ASTM G1-03. La solución empleada como solución decapante fue ácido clorhídrico y hexametiltetramina 0,1 M. Posteriormente, las probetas se enjuagaron con agua destilada, y los residuos de grasa se eliminaron con acetona. Otras probetas fueron limpiadas por chorro de arena o sandblast bajo la norma SSPC-S5, dejándolas en grado metal blanco. Sobre las probetas ya preparadas, se hicieron crecer óxidos, exponiéndolas durante 90 días a la atmósfera urbano-marina de la ciudad de Boca del Rio, Veracruz.

\section{Extracto de Mimosa tenuiflora (convertidor de óxido)}

La extracción de los componentes de la corteza de Mimosa tenuiflora se llevó a cabo por el proceso de maceración dinámica. Este método consistió en utilizar $20 \mathrm{~g}$ de la corteza que fueron colocados en un vaso precipitado de $500 \mathrm{ml}$. Enseguida se prepararon dos formulaciones de convertidor de óxido, conteniendo 150 y $100 \mathrm{ml}$ de etanol (99.5\%) para la primera y segunda, respectivamente. La proporción volumétrica de las dos formulaciones de convertidor de óxido fue de 1:1 (v/v) de 
extracto de Mimosa tenuiflora con una mezcla alcohólica 1:1 (v/v) de isopropanol y terbutanol. Las formulaciones fueron aplicadas sobre los productos de corrosión por medio de un atomizador, para posteriormente dejarse secar durante 24 horas (fig. 1).

a)

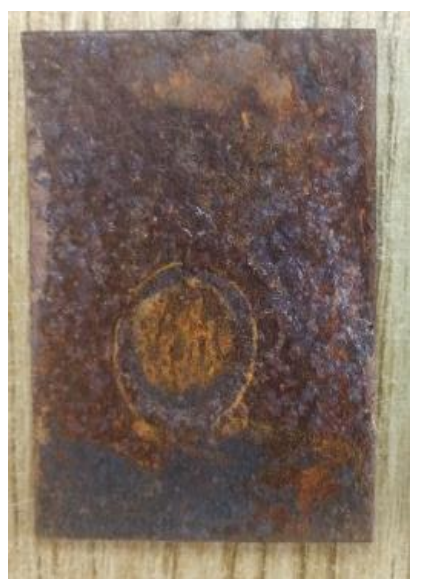

b)

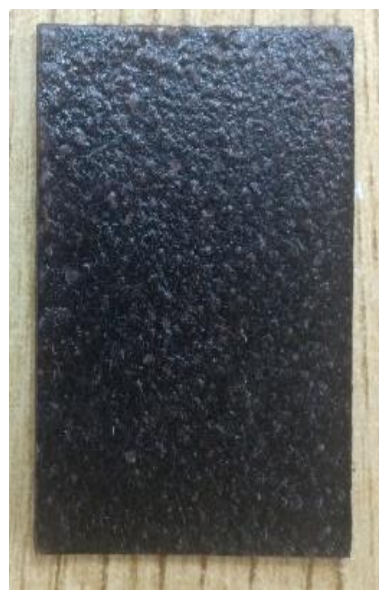

Fig. 1. Probetas con: a) productos de corrosión; y b) película convertida por la formulación del convertidor de óxido.

Fig. 1. Coupons with: a) corrosion products, and b) the converted film by the rust converter formulation.

\section{MEB-EDX}

La observación de los productos de corrosión y el convertidor de óxido se llevó a cabo usando un Microscopio Electrónico de Barrido (MEB) Flexem 1000 (Hitachi), equipado con detectores de electrones secundarios y retrodispersados. Para el análisis químico el MEB, contaba con un detector de Microanálisis de Energía Dispersiva de Rayos X (EDX) Quantax75 (Bruker). Las muestras fueron analizadas sin preparación previa.

\section{Espectroscopía Raman}

La identificación de productos de corrosión se realizó mediante un espectrofotómetro iPlus-Raman equipado con un microscopio (B\&WTEK). Los productos de corrosión se irradiaron con un láser de $785 \mathrm{~nm}$ de longitud de onda. El láser fue ajustado a diferentes potencias, siempre evitando un efecto térmico en las muestras que fueron analizadas.

\section{Difracción de Rayos X (DRX)}

Para la identificación de las fases cristalinas formadas sobre los productos de corrosión de las placas de acero AISI 1018, se realizó en un Difractómetro de Rayos X utilizando un equipo D8 Advance (BRUKER) con diseño DAVINCI y geometría theta-theta que empleaba radiación 
monocromática de tubo de $\mathrm{Cu}(\lambda=1,5406 \AA)$; y un detector de silicio Linxeye operado a: $45 \mathrm{kV}$ y $40 \mathrm{~mA}$. El campo difraccional utilizado fue de 10-120 . La identificación de los difractogramas se realizó mediante el software DIFFRAC. EVA versión 3.1.

\section{Espectroscopía de Impedancia Electroquímica (EIE)}

Para el análisis electroquímico se utilizó una celda convencional de tres electrodos que consistía en un electrodo de trabajo, un electrodo de referencia de $\mathrm{Ag} / \mathrm{AgCl}$ y un contraelectrodo de grafito. El área de análisis fue de $1 \mathrm{~cm}^{2}$, empleando una solución de $\mathrm{NaCl}$ al $3.5 \%$ en peso. Para estabilizar el sistema, se midió el potencial de corrosión ( $\left.\mathrm{E}_{\text {corr }}\right)$ durante $1200 \mathrm{~s}$. Una vez alcanzada la estabilidad, se aplicó la técnica de EIE con los siguientes parámetros de medición: amplitud sinusoidal de $10 \mathrm{mV}$, con un intervalo de barrido de frecuencias de $10 \mathrm{kHz}-1 \mathrm{mHz}$ a partir del $\mathrm{E}_{\text {corr. }}$. La adquisición de los datos electroquímicos se realizó en un potenciostato Gamry Reference 600.

\section{$\underline{\text { Resultados }}$}

Las figs. 2-7 muestran aspectos generales del crecimiento de productos de corrosión en las probetas de acero, tratadas superficialmente con un decapado químico y chorro de arena después de 90 días de exposición a la intemperie. La morfología de los productos de corrosión formados en el acero al carbono puede variar considerablemente; depende de factores como: método de síntesis usado en el laboratorio y las condiciones de exposición atmosférica (Alcántara et al., 2015). La fig. 2, presenta una imagen a 100X de la probeta decapada químicamente, se observa una superficie rugosa y compacta. Con el detector de electrones retrodispersados (BSE; backscattered electron), se puede diferenciar por contraste de numero atómico $(Z)$ la presencia de materiales externos a la capa de productos de corrosión. A 500X, se observó la presencia de porosidades, lo que favorece la penetración de contaminantes que se integran a la capa de humedad para formar un electrolito que acelera el proceso corrosivo.

Así mismo, se pudieron observar estructuras en forma de pétalos de flores, características de la lepidocrocita. Por otra parte, las micrografías obtenidas con el detector de electrones secundarios (SE; secondary electron), presentan a 1000X estructuras globulares (fig. 3a), en cuyo interior se observa una estructura con morfología parecida a una esfera deshidratada llamada wepping. Otros términos descriptivos de este tipo de morfología son perlas amarillas de líquido, glóbulos marrones de líquido y manchas de corrosión delicuescentes en forma de perlas. A 7500X 
(fig. 3b), la morfología adquiere un aspecto de agujas tipo bigote o tipo varilla, similares a las que presenta la goethita (Alcántara et al., 2015).

a)

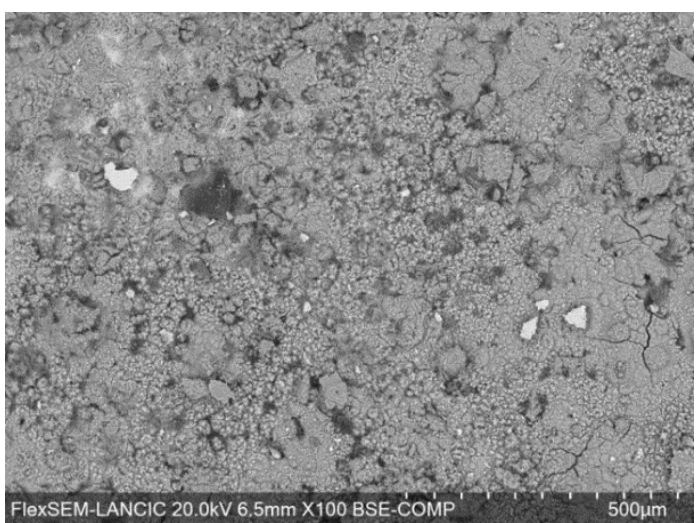

b)

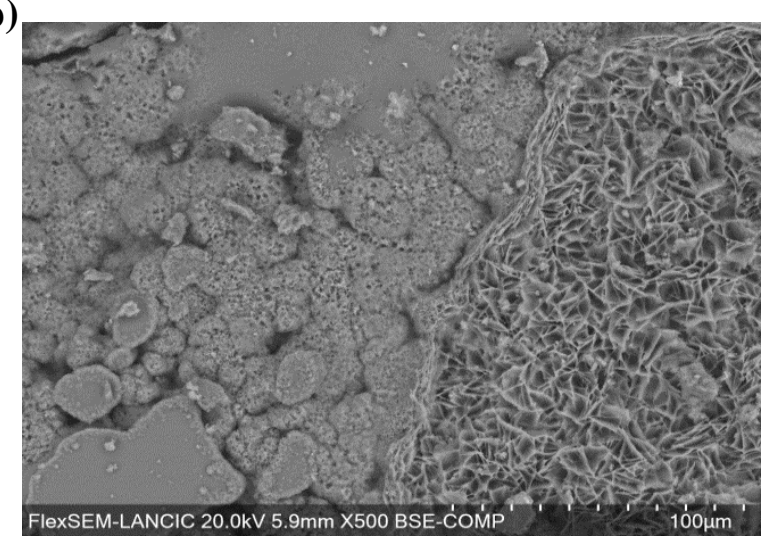

Fig. 2. Productos de corrosión formados en la probeta decapada en aumentos de 100X y 500X obtenidos por el detector de electrones retrodispersados (BSE; por sus siglas en inglés).

Fig. 2. Corrosion products formed on the pickled coupon at 100X and 500X magnifications obtained by backscattered electron detector (BSE).

a)

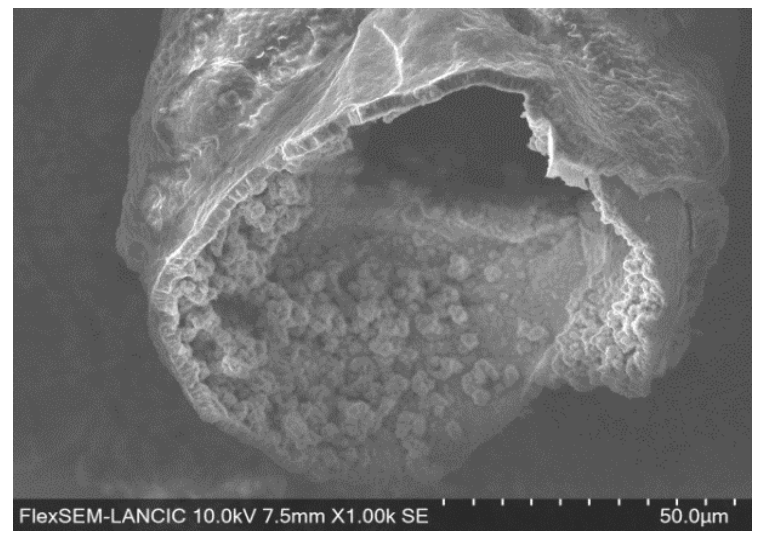

b)

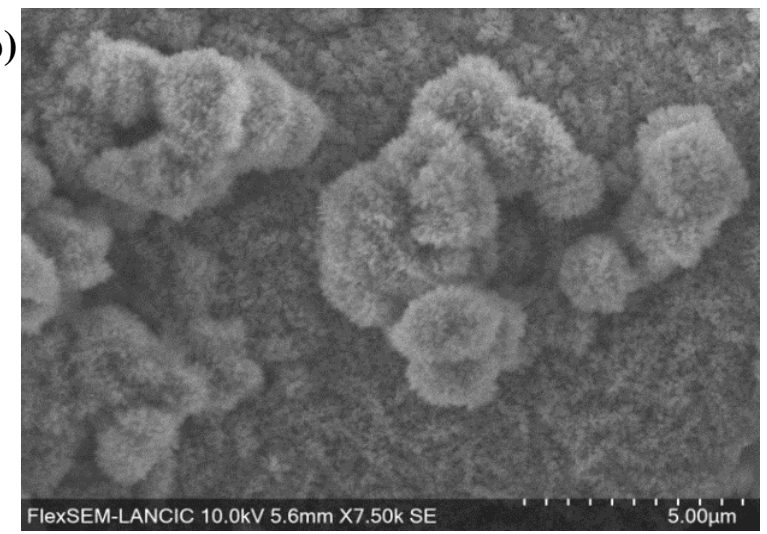

Fig. 3. Productos de corrosión formados en la probeta decapada en aumentos de a) 1000X y b) 7500X, glóbulos. En el interior de los glóbulos se observan estructuras caracteristicas de goethita.

Fig. 3. Corrosion products formed on pickled coupon at a) $1000 \mathrm{X}$ and b) $7500 \mathrm{X}$ magnifications. Wepping and inside the wepping, the typical morphologies of goethite are observed.

La capa de óxido sobre la probeta tratada con chorro de arena presenta a 100X estructuras características de la lepidocrocita (fig. 4a), así como partículas de origen externo incluidas en la capa de productos de corrosión. En la fig. 4b (a 500X), se observan otras estructuras con formas de pétalos de flores y globulares, similares a las presentes en aquellas probetas tratadas con decapado químico. La morfología de la goethita presenta diferentes tipos de estructuras: láminas delgadas, rectas y afiladas, agujas tipo bigote o morfología tipo varilla (Alcántara et al., 2015). Un 
tipo de morfología característica de la goethita semicristalina, con forma de bolas de algodón con láminas afiladas interconectadas por formaciones como nidos, se puede observar en la fig. 5, a 1000 y 5000X (Antunes et al., 2003).

a)

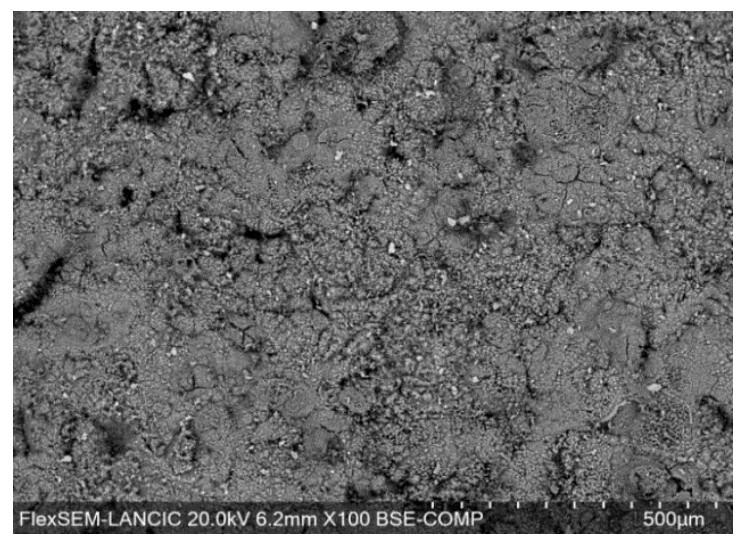

b)

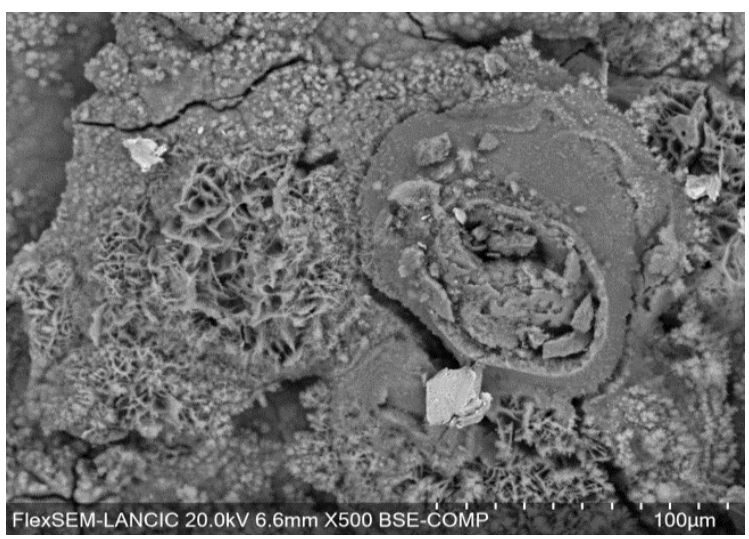

Fig. 4. Productos de corrosión formados en la probeta con chorro de arena para: a) 100X; y b) 500X. Se observan morfologías de petalos de flores y globulos.

Fig. 4. Corrosion products formed on the sandblasted coupon sandblasted coupon at: a) 100X, and b) 500X. Morphologies flower petals and wepping.

a)

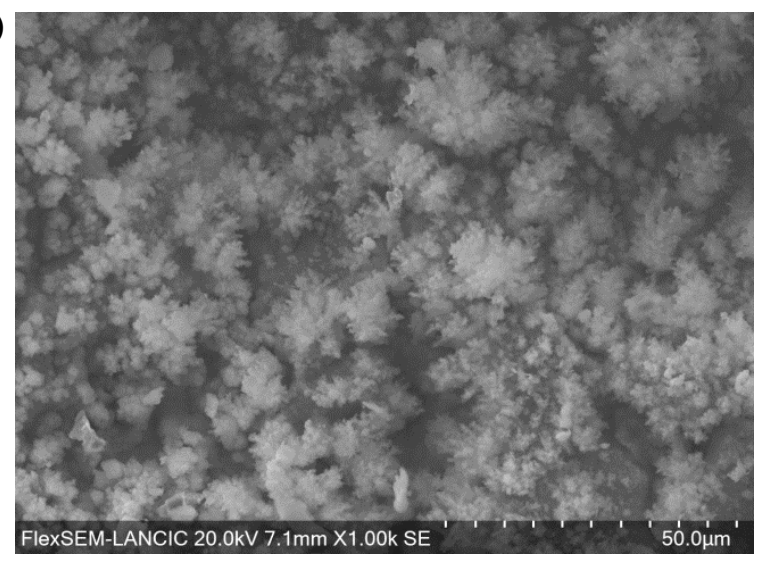

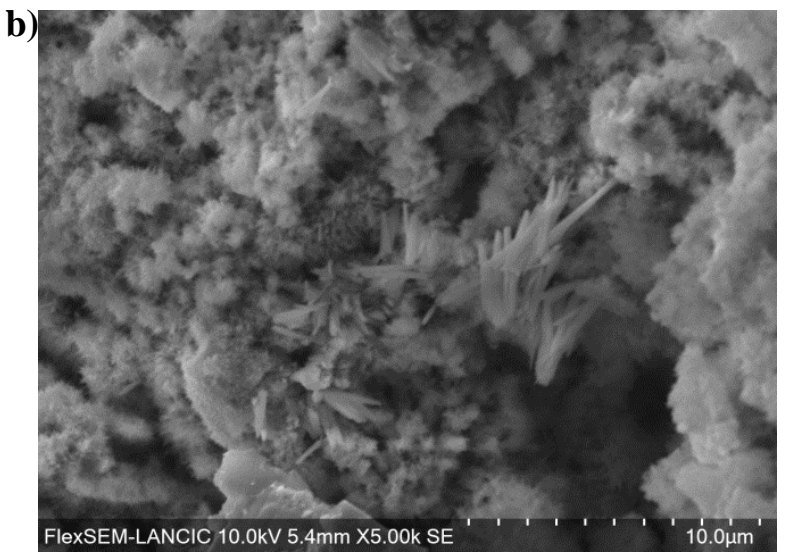

Fig. 5. Morfología tipica de bolas de algodón y bigote de la goethita formadas en la probeta con chorro de arena: a) 1000X; y b) 5000X.

Fig. 5. Typical morphology of cotton balls and whisker-like morphology of goethite formed on sandblasted coupon: a) 1000X; y b) 5000X.

Otra propiedad importante de los productos de corrosión, que puede influir en el comportamiento del proceso corrosivo, es la permeabilidad de la capa de óxidos, es decir, su porosidad a especies iónicas (Dillman et al., 2004). En este sentido, elementos exógenos provenientes del medio ambiente como $\mathrm{C}, \mathrm{Na}, \mathrm{Al}, \mathrm{Si}, \mathrm{Cl}$ y $\mathrm{Ca}$ (tabla 1 ), pueden quedar atrapados en la capa de óxido y en algunos casos incrementar la conductividad iónica del electrolíto. 
Tabla 1. Composición química elemental de la capa de productos de corrosión obtenida mediante EDS. D1 y D2: probetas decapadas; S1 and S2: probetas limpiadas con chorro de arena; - no detectado.

Table 1. Elemental chemical composition of corrosion products layer. D1 y D2: pickled coupons, S1 y S2: sandblasted coupons, - undetected.

\begin{tabular}{|c|c|c|c|c|c|c|c|c|}
\hline \multirow{2}{*}{ Muestra } & \multicolumn{8}{|c|}{ Composición química elemental (\% peso) } \\
\cline { 2 - 9 } & $\mathrm{C}$ & $\mathrm{O}$ & $\mathrm{Na}$ & $\mathrm{Al}$ & $\mathrm{Si}$ & $\mathrm{Cl}$ & $\mathrm{Ca}$ & $\mathrm{Fe}$ \\
\hline D1 & 4.05 & 22.94 & 1.98 & - & - & 0.78 & 0.30 & 69.96 \\
\hline D2 & 4.34 & 30.19 & - & - & 0.19 & - & - & 65.07 \\
\hline S1 & 4.61 & 27.89 & 2.07 & - & - & 0.12 & 0.28 & 65.02 \\
\hline S2 & 3.84 & 29.09 & - & 0.38 & 0.88 & - & 0.19 & 65.63 \\
\hline
\end{tabular}

En las figs. 6-9, se presentan las micrografías correspondientes a la película convertida por las dos formulaciones de convertidor de óxido sobre productos de corrosión del acero al carbono con diferente tratamiento superficial. La morfología de la película convertida es amorfa, compacta y uniforme, además se observó la presencia de algunas microgrietas. También, se pueden observar abultamientos y zonas brillantes que corresponden a zonas del metal base donde el Fe no pudo quelatarse con el convertidor de óxido (Ross y Francis 1978; Barrero et al., 2001; Collazo et al., 2008; Zhao et al., 2014; Feng et al., 2017; Xu et al., 2019).

Tabla 2. Composición química elemental de la película convertida por accion de las dos formulaciones de convertidor de óxido; D1 y D2: probetas decapada; S1 y S2: probetas con chorro de arena, - no detectado.

Table 2. Elemental chemical composition of the film converter by action of the two oxide converter formulations, D1 and D2: pickled coupon, S1 and S2: sandblasted coupon, - undetected.

\begin{tabular}{|c|c|c|c|c|c|c|}
\hline \multirow{2}{*}{ Muestra } & \multicolumn{6}{|c|}{ Composición química elemental (\% peso) } \\
\cline { 2 - 7 } & $\mathrm{C}$ & $\mathrm{O}$ & $\mathrm{Na}$ & $\mathrm{K}$ & $\mathrm{Cl}$ & $\mathrm{Fe}$ \\
\hline D1 & 59.78 & 45.07 & - & - & 0.48 & 4.67 \\
\hline D2 & 64.78 & 33.65 & - & 0.23 & 0.12 & 1.22 \\
\hline S1 & 60.70 & 30.52 & 3.04 & - & 0.77 & 4.98 \\
\hline S2 & 68.17 & 31.06 & - & - & - & 0.77 \\
\hline
\end{tabular}


a)

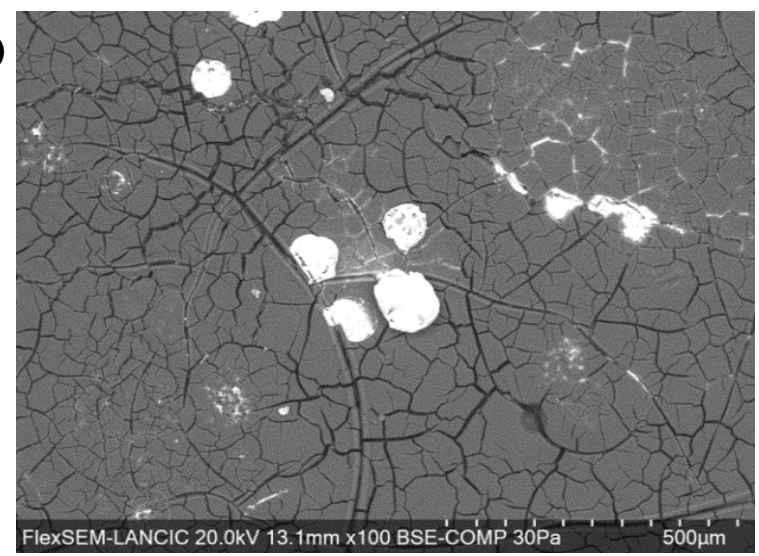

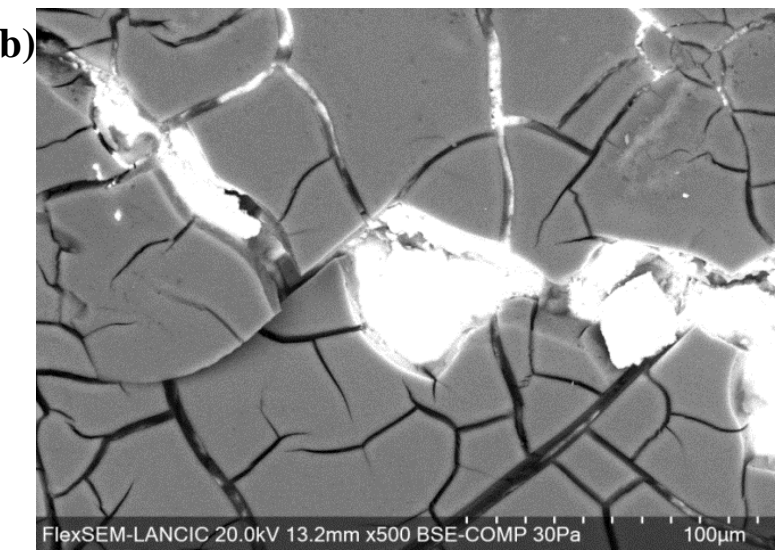

Fig. 6. Superficie convertida con la formulación que contiene $150 \mathrm{ml}$ de etanol como solvente sobre la probeta tratada superficialmente con decapado químico: a) 100X; y b) 500X.

Fig. 6. Surface converted by the formulation containing $150 \mathrm{ml}$ of ethanol as solvent over the coupons with chemical pickling treatment at: a) 100X, and b) 500X.

a)

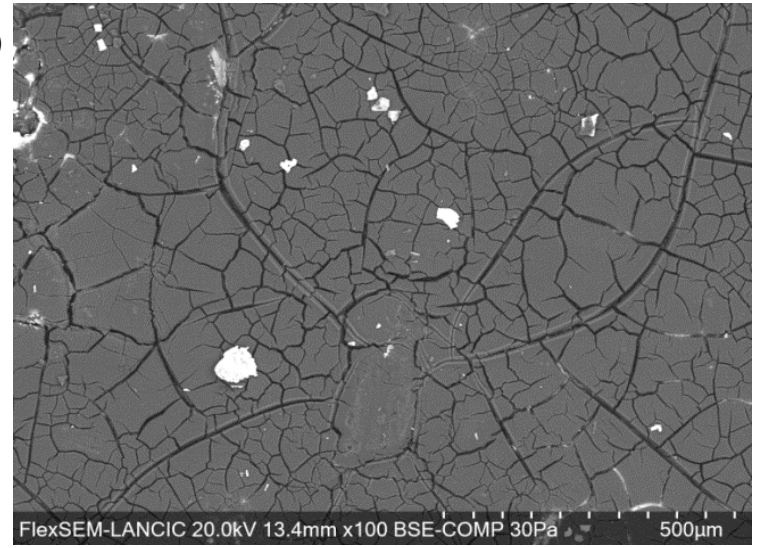

b)

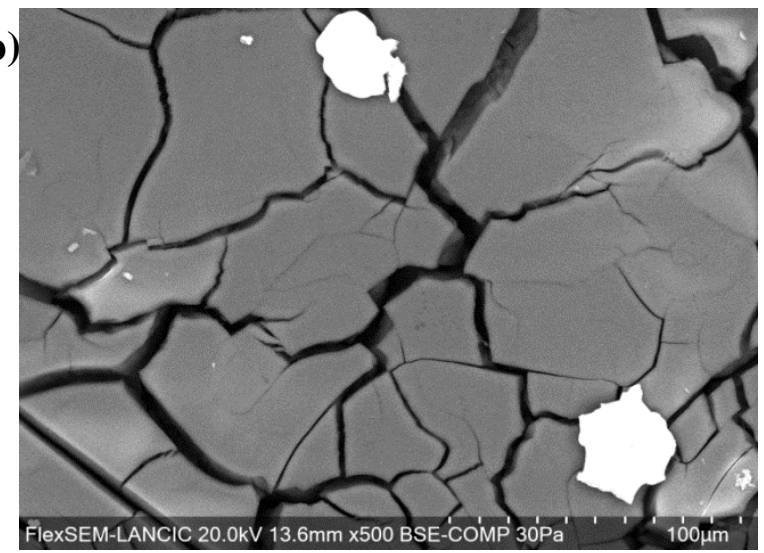

Fig. 7. Superficie convertida con la formulación que contiene $150 \mathrm{ml}$ de etanol como solvente sobre la probeta tratada superficialmente con chorro de arena: a) 100X; y b) 500X.

Fig. 7. Surface converted by the formulation containing $150 \mathrm{ml}$ of ethanol as solvent over the coupons with sandblasting surface treatment at: a) $100 \mathrm{X}$, and b) $500 \mathrm{X}$. 
a)

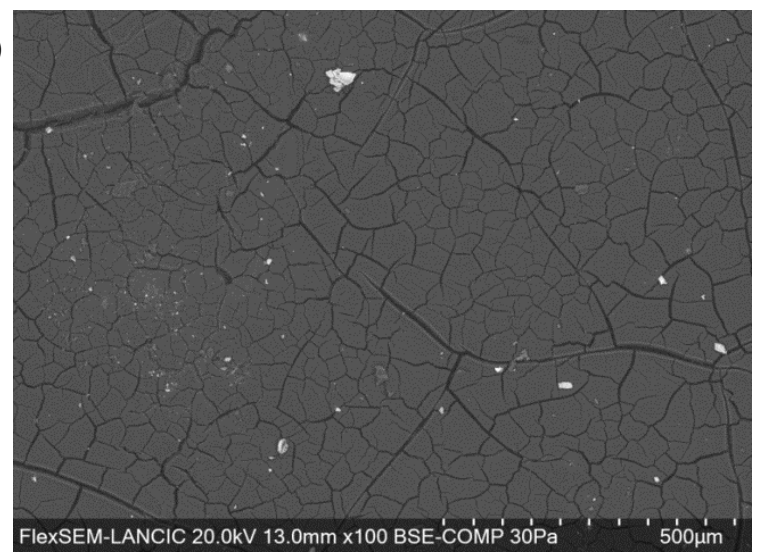

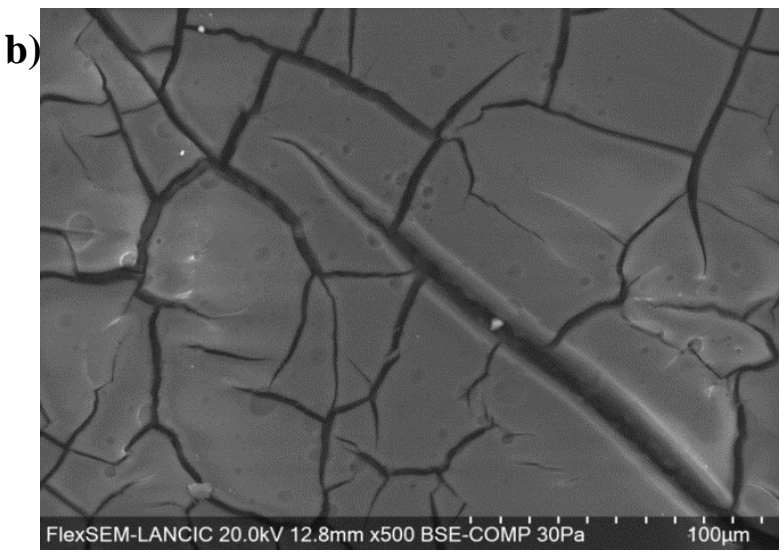

Fig. 8. Superficie convertida con la formulación que contiene $100 \mathrm{ml}$ de etanol como solvente sobre la probeta tratada superficialmente con decapado químico: a) 100X; y b) 500X.

Fig. 8. Surface converted by the formulation containing $100 \mathrm{ml}$ of ethanol as solvent over the coupons with chemical pickling treatment at: a) $100 \mathrm{X}$, and b) 500X.

a)

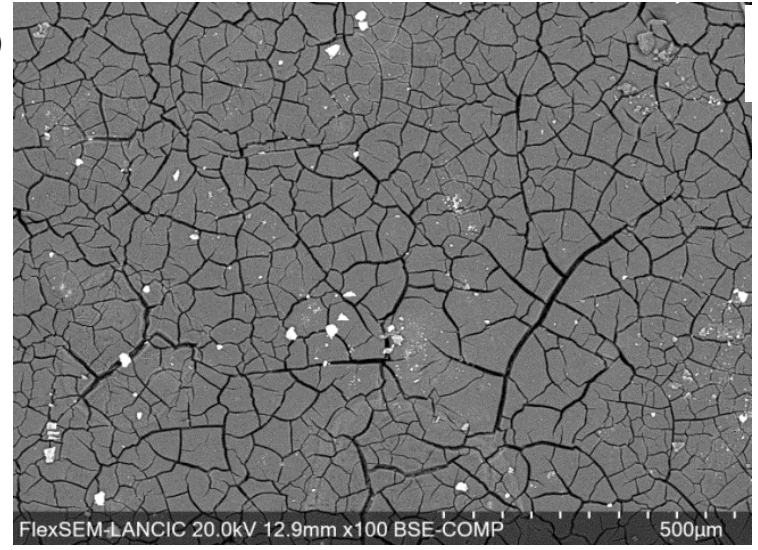

b)

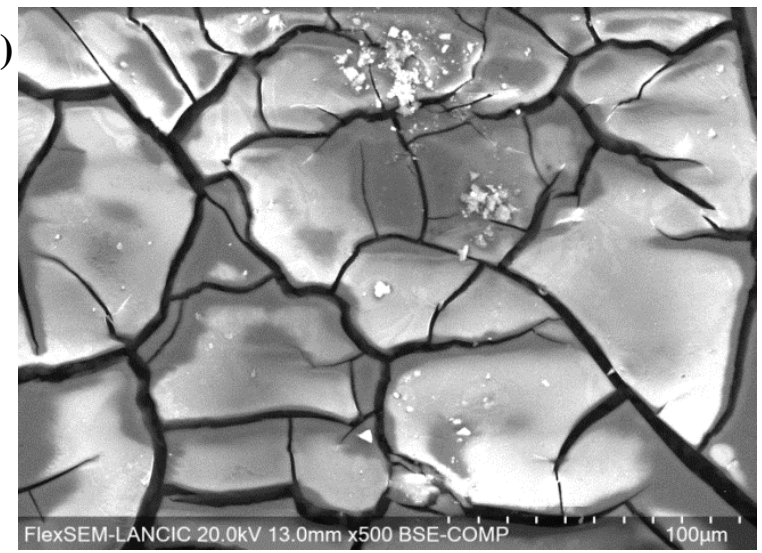

Fig. 9. Superficie convertida con la formulación que contiene $100 \mathrm{ml}$ de etanol como solvente sobre la probeta tratada superficialmente con chorro de arena: a) 100X; y b) 500X.

Fig. 9. Surface converted by the formulation containing $100 \mathrm{ml}$ of ethanol as solvent on the sandblasted coupons surface at: a) 100X, and b) 500X.

Los espectros Raman de los productos de corrosión formados después de 90 días de exposición a la intemperie se presentan en las Figs. 10a y 10b. La lepidocrocita presenta dos picos característicos en 532 y $640 \mathrm{~cm}^{-1}$. La fase principal identificada fue la goethita con una banda de flexión asimétrica en $400 \mathrm{~cm}^{-1}$ atribuido a enlaces Fe-OH un pico de mayor intensidad en $242 \mathrm{~cm}^{-1}$. Otros bandas relacionadas a la fase de goethita se encontraron en 220, 288 y $685 \mathrm{~cm}^{-1}$ (de Faria et al., 1997; Legodi y de Waal, 2007; Sancy et al., 2010; de la Fuente et al., 2016). 
Por su parte, la hematita tuvo picos en 415 y $600 \mathrm{~cm}^{-1}$ relacionados a enlaces $\mathrm{Fe}-\mathrm{O}$ de estiramiento y flexión simétrica respectivamente (Legodi \& de Waal, 2007; Sancy et al., 2010; Zhang et al., 2011; Criado et al., 2015; Ramanaidou et al., 2015; de la Fuente et al., 2016).
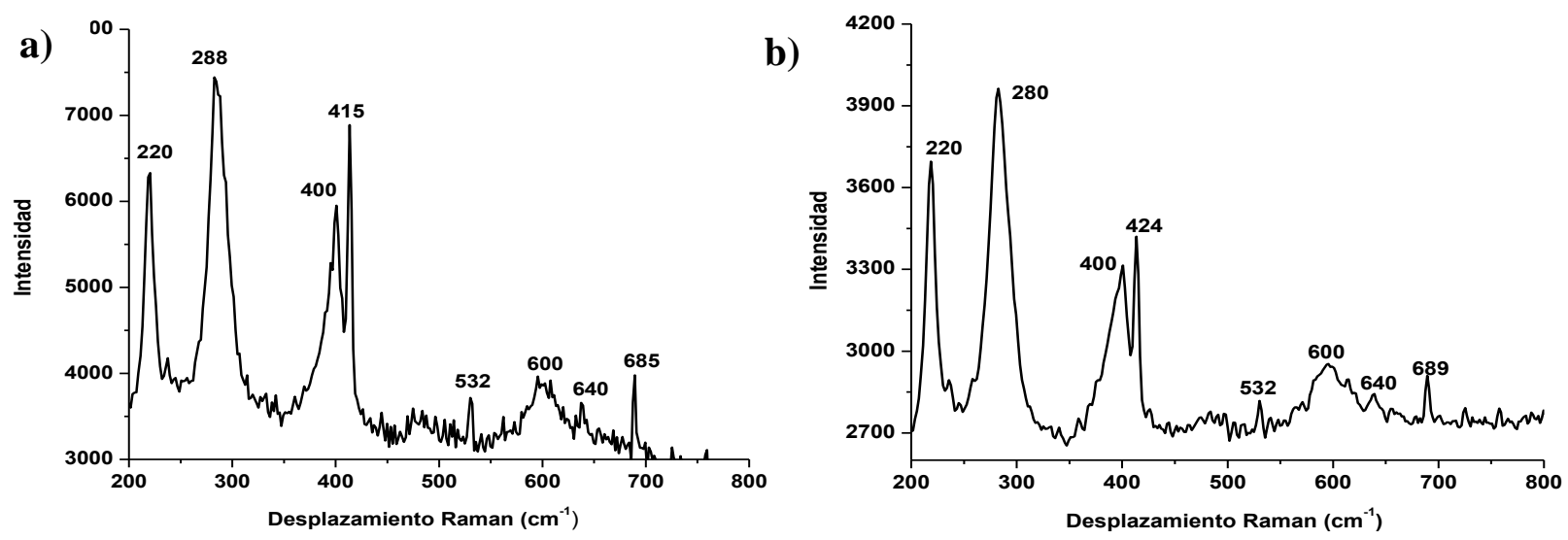

Fig. 10. Espectro Raman de los productos de corrosión de la probeta tratada con: a) decapado químico y con b) chorro de arena.

Fig. 10. Raman spectrum of corrosion products on: a) pickled coupon and b) sandblasted coupon.

La composición de los productos de corrosión formados sobre el acero tratado por decapado químico y chorro de arena presentaron las mismas fases cristalinas. Esto último, era de esperarse ya que su formación ocurrió en mismas condiciones de exposición ambiental. En este sentido, un parámetro importante en el anclaje eficientemente del convertidor de óxido es su adherencia de la capa de óxidos sobre la superficie tratada. Las fases cristalinas identificadas mediante DRX (fig. 11) fueron: lepidocrocita $(\gamma-\mathrm{FeOOH})$ y goethita $(\boldsymbol{\alpha}-\mathrm{FeOOH})$. Además, se identificó la fase de hierro perteneciente al sustrato metálico. Estas fases cristalinas son productos de corrosión característicos de aceros expuestos a la intemperie (Dillman et al., 2004; Alcántara et al., 2015; de la Fuente et al., 2016). 

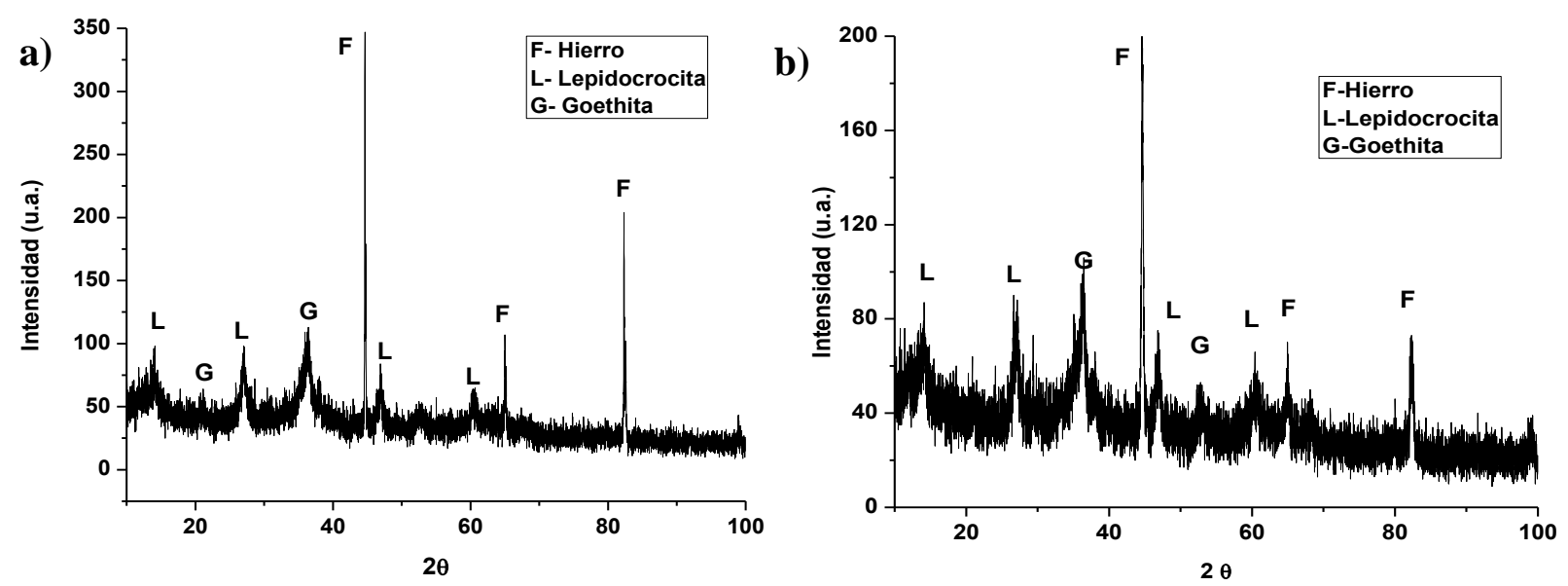

Fig. 11. Difractograma de los productos de corrosión de la probeta tratada con: a) decapado químico; y b) chorro de arena. Fases cristalinas identificadas: F: hierro, L: lepidocrocita, G: goethita.

Fig. 11. Diffractograms corresponding to corrosion products from: a) pickled, and b) sandblasted coupons. Identified crystalline phase: F: iron, L: lepidocrocite, G: goethite.

En la fig. 12, se presentan los diagramas de Nyquist correspondientes a las probetas. En las probetas tratadas con decapado químico (fig. 12a) se encontraron valores de impedancia de aproximadamente $90 \Omega . \mathrm{cm}^{2}$. En cambio, en aquellas tratadas con chorro de arena (fig. 12b) se observó que la capa de productos de corrosión presentaba valores de impedancia mayores a los 140 $\Omega . \mathrm{cm}^{2}$. En los diagramas de Nyquist de las figs. 13 y 14, se presenta el comportamiento del convertidor de óxido sobre las probetas de acero con diferente tratamiento superficial. Cabe resaltar que las formulaciones del convertidor de óxido, presentaron un mejor comportamiento electroquímico en las probetas tratadas con chorro de arena (figs. 13a y 13b). Por otro lado, los resultados con las dos formulaciones parecen indicar que el empleo de $100 \mathrm{ml}$ de etanol, proporciona una menor extracción del convertidor de óxido (figs. 14a y 14b). En todos los diagramas de Nyquist se observaron pendientes cercanas a los $45^{\circ}$ en frecuencias bajas o intermedias que sugieren la presencia de un proceso de difusión por transferencia de masa dentro de la capa de productos de corrosión, así como en la película convertida (Feliu et al., 1993). 
Protección anticorrosiva de un convertidor de óxido natural (Mimosa tenuiflora) aplicado sobre productos de corrosión de un acero AISI 1018
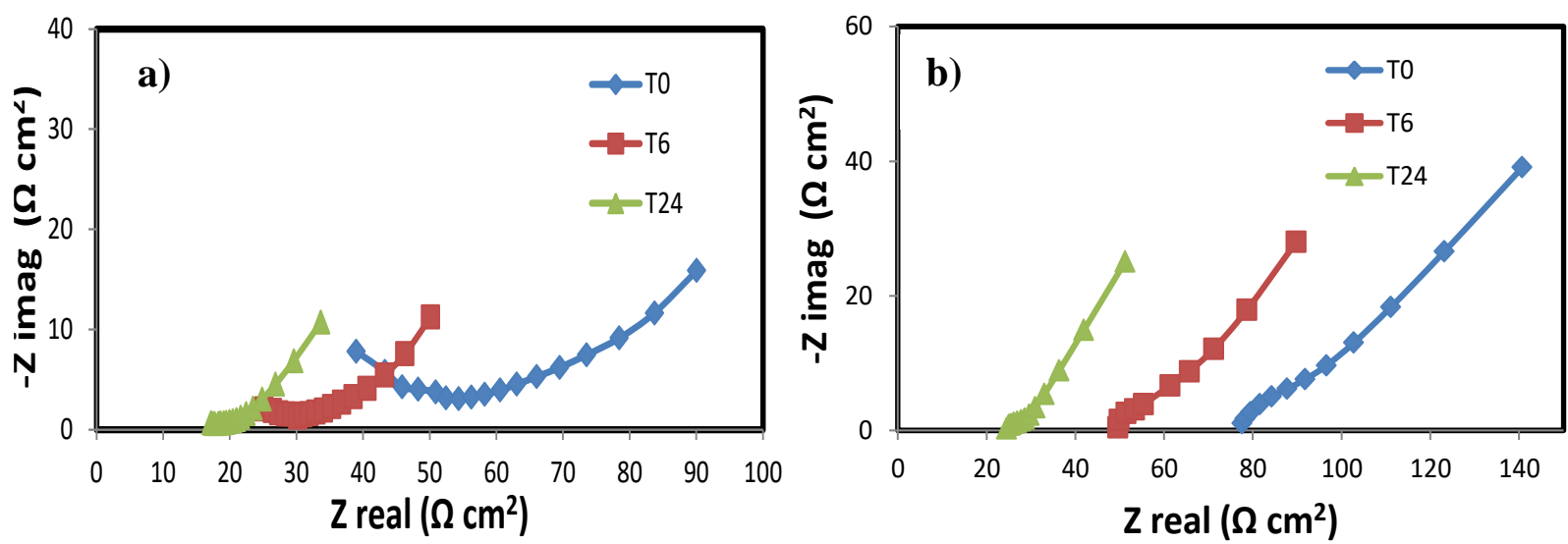

Fig. 12. Diagramas de Nyquist de los productos de corrosión de la probeta: a) decapada; y con b) chorro de arena a diferentes tiempos de exposición en una solución de $\mathrm{NaCl}$ al $3.5 \%$

Fig. 12. Nyquist diagram of corrosion products of: a) pickled, and b) sandblasted coupon at different exposure times in $3.5 \% \mathrm{NaCl}$ solution.
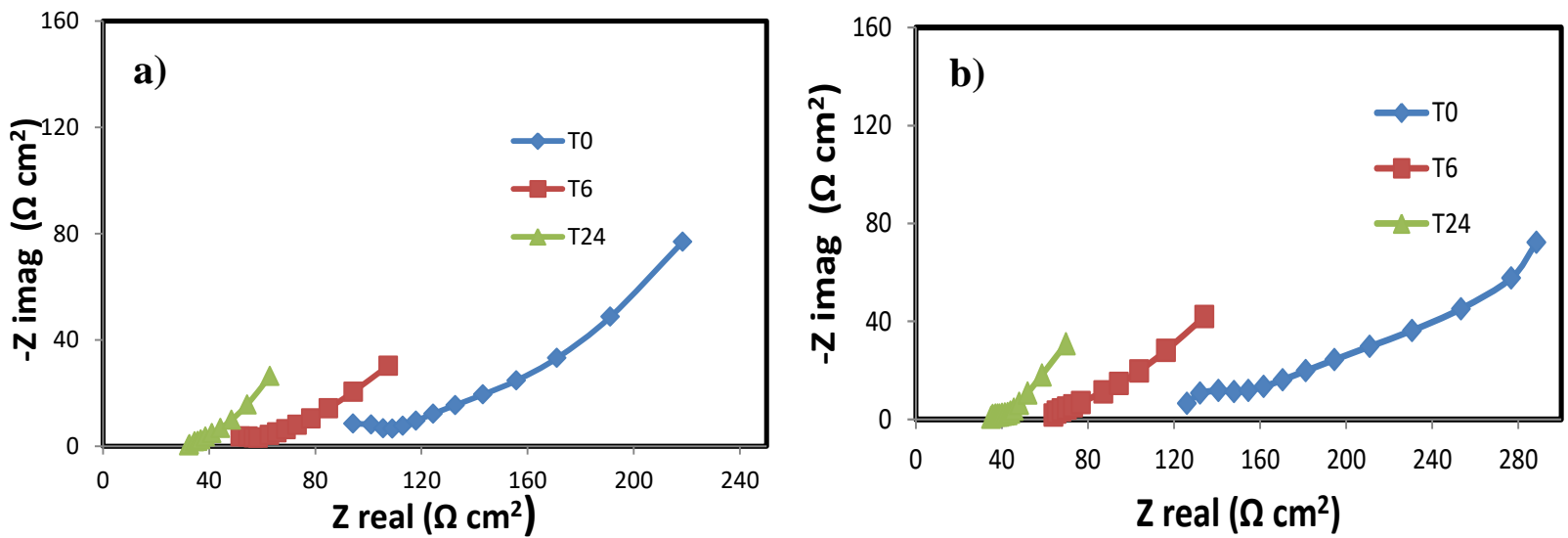

Fig. 13. Diagrama de Nyquist de la película convertida (formulación con $150 \mathrm{ml}$ de etanol) de la probeta: a) decapada; y b) chorro de arena a diferentes tiempos de exposición en una solución de $\mathrm{NaCl}$ al $3.5 \%$.

Fig. 13. Nyquist diagram of the converted film (formulation with $150 \mathrm{ml}$ of ethanol) in: a) pickled, and b) sandblasted coupon at different exposure time in $3.5 \% \mathrm{NaCl}$ solution.
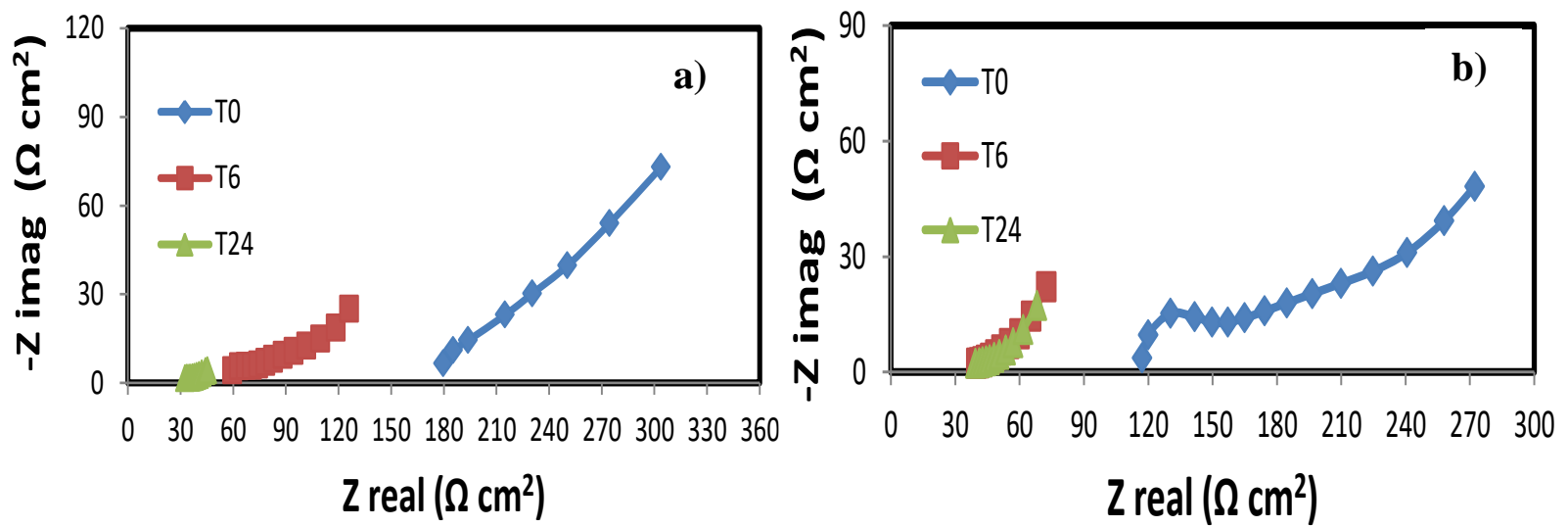

Fig. 14. Diagramas de Nyquist de la película convertida (formulación con 100ml de etanol) de la: a) placa decapada; y con b) chorro de arena a diferentes tiempos de exposición en una solución de $\mathrm{NaCl}$ al $3.5 \%$.

Fig. 14. Nyquist diagram of film converter (formulation with $100 \mathrm{ml}$ ethanol) of: a) pickled, y b) sandblasted plated at different exposure time in $3.5 \% \mathrm{NaCl}$ solution. 


\section{Discusión y conclusiones}

Se sabe que la alteración superficial de un metal puede conducir a mejoras en sus propiedades mecánicas, así como a una mejor resistencia a la corrosión (Ding y Poursaee, 2017). Esto ocurrió durante este estudio con las probetas de acero cuando se trataron con el chorro de arena- El chorro de arena permitió obtener una superficie con mayor rugosidad, debido a la deformación plástica local que se genera en el metal permitiendo mejorar el comportamiento mecánico y la adherencia de los productos de corrosión (Gen et al., 2015). Por su parte, el tratamiento superficial por decapado químico crea una superficie menos rugosa de menor capacidad adherente.

La capa de productos de corrosión está compuesta principalmente por goethita y lepidocrocita, fases cristalinas comunes formadas en aceros expuestos a la intemperie. Además, mediante el uso de Espectroscopía Raman se identificó una tercera fase cristalina, la hematita $\left(\mathrm{Fe}_{2} \mathrm{O}_{3}\right)$. La composición química elemental de los productos de corrosión indica la presencia de Fe y $\mathrm{O}$ principalmente. Los otros elementos identificados se consideraron externos a la capa de óxido. El carbono (C), puede tener su origen en depósitos ambientales de materia orgánica, mientras que el $\mathrm{Al}$ y Si, se encuentran en partículas de polvo de aluminosilicatos. Por su parte, el calcio $(\mathrm{Ca})$ probablemente se debe a la presencia de partículas de polvo ricas en calcita $\left(\mathrm{CaCO}_{3}\right)$, normalmente originados en suelos locales, mientras que $\mathrm{Cl}^{-}$y Na, tienen su origen en aerosoles marinos característicos de la atmósfera marina de la ciudad de Boca del río. Esta capa de productos de corrosión no varía en su composición química o estructural, sin embargo, el tipo de morfología y su distribución sobre la superficie puede repercutir en sus propiedades anticorrosivas. Lo anterior, pudo observarse en los resultados obtenidos mediante el empleo de EIE, los cuales muestran diferencias en sus valores de impedancia (Zreal). Esto es consecuencia del tratamiento por chorro de arena, pues provee un mejor anclaje a la capa de óxido, lo que permite obtener una capa adherente de mayor eficacia para la aplicación de un convertidor de óxido como un recubrimiento primario.

Los resultados por EDS mostraron que las capas de productos convertidos con las distintas formulaciones tienen un bajo contenido en $\mathrm{Fe}(<5 \%$ en peso), indicativo de la transformación de los productos de corrosión en quelatos de hierro, que se forman por los polifenoles del extracto de Mimosa tenuiflora. El Fe remanente puede deberse a la fase de goethita la cual reacciona a menor velocidad para formar complejos con los componentes del extracto. Por otra parte, se demostró que 
utilizar una menor cantidad de solvente de extracción para la formulación del convertidor de óxido, proporciona una mejor protección anticorrosiva.

La película convertida de apariencia craquelada y compacta se degradó en presencia de la solución de iones cloruro después de 24 horas de exposición. Esto se debió principalmente al ingreso de estos iones a la interfaz metal/película convertida, ya que su actividad catalítica inicialmente provoca su ruptura, para posteriormente ingresar a la superficie metálica y provocar hendiduras. Otro problema que causan los iones cloruros es la disminución del pH, por formación gas de $\mathrm{HCl}$. La ruptura de la película facilita la difusión de oxígeno hacia la superficie metálica aumentando la reacción anódica, y por ende, aumentando también la reacción catódica hasta alcanzar el equilibrio.

La capa superficial de óxido es necesaria para facilitar la adherencia del recubrimiento, aunque también puede ser la causa de su desprendimiento o ruptura (Ross y Francis, 1978). Desafortunadamente, las condiciones atmosféricas de un ambiente marino o con alto contenido de iones cloruro no son adecuadas para el buen funcionamiento de los convertidores de óxido, ya que su degradación resultará inevitable y su permanencia sobre la superficie será momentáneo.

En conclusión, la preparación superficial por chorro de arena es adecuada para obtener un mejor anclaje de la capa de óxidos y así mismo del convertidor de óxido. El convertidor de óxido obtenido del extracto de Mimosa tenuiflora aumenta las propiedades anticorrosivas de la capa de óxido. Sin embargo, el empleo de este convertidor de óxido está limitado solo a atmósferas interiores o con bajo contenido de iones cloruro. La aplicación de este convertidor de óxido natural puede ser utilizada en el ámbito industrial y el patrimonio cultural metálico, únicamente para artefactos de hierro.

\section{Agradecimientos}

Se agradece al Consejo Nacional de Ciencia y Tecnología, México (CONACYT) y al Programa de Doctorado en Materiales y Nanociencia del Centro de Investigación en Micro y Nanotecnología (MICRONA) de la Universidad Veracruzana. De igual manera, se agradece al Laboratorio Nacional de Ciencias para la Investigación y Conservación del Patrimonio Cultural, LANCICCICORR de la Universidad Autónoma de Campeche por el uso de los equipos de caracterización de materiales (Proyecto CONACYT-LANCIC LN314886), y al Dr. Ildefonso Pech, por su 
asistencia técnica durante los análisis MEB/EDX. Por último, se reconoce al Instituto de Ingeniería de la Universidad Veracruzana, donde se permitió realizar el análisis electroquímico.

\section{$\underline{\text { Referencias }}$}

Alcántara, J., Chico, B., Díaz, I., De la Fuente, D., y Morcillo, M. (2015). Airborne chloride deposit and its effect on marine atmospheric corrosion of mild steel. Corrosion Science, 97, 74-88. DOI: $\underline{\text { https://doi.org/10.1016/j.corsci.2015.04.015 }}$

Antunes, R. A., Costa, I., y Faria, D. L. A. D. (2003). Characterization of corrosion products formed on steels in the first months of atmospheric exposure. Materials Research, 6(3), 403-408. DOI: https://doi.org/10.1590/S1516-14392003000300015

Arceo Gómez, D. E., Aguilar, J. C., Reyes, J., Galván Martínez, R., \& Orozco-Cruz, R. (2019). Electrochemical Analysis of a $\mathrm{SiO}_{2}$ Film on Alternative Rust Converter to Preserve Ferrous Alloys in Historical Heritage. ECS Transactions, 94(1), 229. DOI: https://doi.org/10.1149/09401.0229ecst

ASTM Standard A48/A48M-03 (Reapproved 2012). (2012). Standard Specification for Gray Iron Castings.

ASTM G1-03. (2017). Standard Practice for Preparing, Cleaning, and Evaluating Corrosion Test Specimens.

Barrero, C. A., Ocampo, L. M., y Arroyave, C. E. (2001). Possible improvements in the action of some rust converters. Corrosion Science, 43(6), 1003-1018. DOI: https://doi.org/10.1016/S0010-938X(00)00139-6

Božović, S., Gvozdanović, T., Kraš, A., Grudić, V., Kurajica, S., y Martínez, S. (2020). Rust layer growth and modification by a tannin-based mixture for lowering steel corrosion rates in neutral saline solution. Corrosion Engineering, Science and Technology, 55(5), 372-380. DOI: https://doi.org/10.1080/1478422X.2020.1734739

Byrne, C., D’Alessandro, O., Selmi, G. J., Romagnoli, R., y Deyá, C. (2019). Primers based on tara and quebracho tannins for poorly prepared steel surfaces. Progress in Organic Coatings, 130, 244-250. DOI: https://doi.org/10.1016/j.porgcoat.2019.02.003

Collazo, A., Nóvoa, X. R., Pérez, C., y Puga, B. (2008). EIS study of the rust converter effectiveness under different conditions. Electrochimica Acta, 53(25), 7565-7574. DOI: https://doi.org/10.1016/j.electacta.2007.11.078 
Chaparro, W. A. A., Ruiz, J. H. B., y Rivera, W. G. (2011). Identificación de productos de corrosión en aceros embebidos en concretos alternativos inmersos en $\mathrm{NaCl} 3.5 \%$. Avances: Investigacion en Ingeniería, 8(1), 32-39. Recuperado de: Identificación de productos de corrosión en aceros embebidos en concretos alternativos inmersos en $\mathrm{NaCl} 3.5 \%$ - Dialnet (unirioja.es)

Criado, M., Martínez-Ramírez, S., y Bastidas, J. M. (2015). A Raman spectroscopy study of steel corrosion products in activated fly ash mortar containing chlorides. Construction and Building Materials, 96, 383-390. DOI: https://doi.org/10.1016/j.conbuildmat.2015.08.034

De Faria, D. L., Venâncio Silva, S., y De Oliveira, M. T. (1997). Raman microspectroscopy of some iron oxides and oxyhydroxides. Journal of Raman Spectroscopy, 28(11), 873-878. DOI:https://doi.org/10.1002/(SICI)1097-4555(199711)28:11\%3C873::AIDJRS177\%3E3.0.CO;2-B

De la Fuente, D., Alcántara, J., Chico, B., Díaz, I., Jiménez, J. A., y Morcillo, M. (2016). Characterisation of rust surfaces formed on mild steel exposed to marine atmospheres using XRD and SEM/Micro-Raman techniques. Corrosion Science, 110, 253-264. doi: https://doi.org/10.1016/j.corsci.2016.04.034

Ding, L., y Poursaee, A. (2017). The impact of sandblasting as a surface modification method on the corrosion behavior of steels in simulated concrete pore solution. Construction and Building Materials, 157, 591-599. DOI: https://doi.org/10.1016/j.conbuildmat.2017.09.140 Dillmann, P., Mazaudier, F., y Hœrlé, S. (2004). Advances in understanding atmospheric corrosion of iron. I. Rust characterisation of ancient ferrous artefacts exposed to indoor atmospheric corrosion. Corrosion Science, 46(6), 1401-1429. DOI: https://doi.org/10.1016/j.corsci.2003.09.027

Favre, M., \& Landolt, D. (1993). The influence of gallic acid on the reduction of rust on painted steel surfaces. Corrosion Science, 34(9), 1481-1494. DOI: https://doi.org/10.1016/0010938X(93)90243-A

Favre, M., Landolt, D., Hoffman, K., \& Stratmann, M. (1998). Influence of gallic acid on the phase transformation in iron oxide layers below organic coatings studied with Moessbauer spectroscopy. Corrosion Science, 40(4-5), 793-803. DOI: https://doi.org/10.1016/S0010938X(98)00001-8 
Feng, Y., Ge, S., Li, J., Li, S., Zhang, H., Chen, Y., y Guo, Z. (2017). Synthesis of 3, 4, 5trihydroxy-2-[(hydroxyimino) methyl] benzoic acid as a novel rust converter. Green Chemistry Letters and Reviews, 10(4), 455-461. DOI: https://doi.org/10.1080/17518253.2017.1400590

Feliu, S., Galván, J. C., Feliu Jr., S., Bastidas, J. M., Simancas, J., Morcillo, M., y Almeida, E. M. (1993). An electrochemical impedance study of the behaviour of some pretreatments applied to rusted steel surfaces. Corrosion Science, 35(5-8), 1351-1358. DOI: https://doi.org/10.1016/0010-938X(93)90357-M

Geng, S., Sun, J., y Guo, L. (2015). Effect of sandblasting and subsequent acid pickling and passivation on the microstructure and corrosion behavior of $316 \mathrm{~L}$ stainless steel. Materials \& Design, 88, 1-7. DOI: https://doi.org/10.1016/j.matdes.2015.08.113

Jaramillo, A. F., Montoya, L. F., Prabhakar, J. M., Sanhueza, J. P., Fernández, K., Rohwerder, M., y Melendrez, M. F. (2019). Formulation of a multifunctional coating based on polyphenols extracted from the Pine radiata bark and functionalized zinc oxide nanoparticles: Evaluation of hydrophobic and anticorrosive properties. Progress in Organic Coatings, 135, 191-204. DOI: https://doi.org/10.1016/j.porgcoat.2019.06.011

Legodi, M. A., y de Waal, D. (2007). The preparation of magnetite, goethite, hematite and maghemite of pigment quality from mill scale iron waste. Dyes and Pigments, 74(1), 161168. DOI: https://doi.org/10.1016/j.dyepig.2006.01.038

Li, J., Ge, S., Wang, J., Du, H., Song, K., Fei, Z., ... y Guo, Z. (2018). Water-based rust converter and its polymer composites for surface anticorrosion. Colloids and Surfaces A: Physicochemical and Engineering Aspects, 537, 334-342. DOI: https://doi.org/10.1016/j.colsurfa.2017.10.041

Matamala, G., Smeltzer, W., y Droguett, G. (1994). Use of tannin anticorrosive reaction primer to improve traditional coating systems. Corrosion, 50(4), 270-275. DOI: https://doi.org/10.5006/1.3294333

Morteo-Flores, A. O., Galván-Martínez, R., Fernández-Gómez, I., y Orozco-Cruz, R. (2018). Assessing Mimosa Tenuiflora Extract as Rust Converter on Iron Corrosion Products. ECS Transactions, 84(1), 341. DOI: https://doi.org/10.1149/08401.0341ecst 
Ocampo, L. M., Margarit, I. C. P., Mattos, O. R., Córdoba-de-Torresi, S. I., y Fragata, F. L. (2004). Performance of rust converter based in phosphoric and tannic acids. Corrosion Science, 46(6), 1515-1525. DOI: https://doi.org/10.1016/j.corsci.2003.09.021

Ramanaidou, E. M. I. C., Wells, M., Lau, I., y Laukamp, C. (2015). Characterization of iron ore by visible and infrared reflectance and, Raman spectroscopies. In Iron ore (pp. 191-228). Woodhead Publishing. DOI: https://doi.org/10.1016/B978-1-78242-156-6.00006-X

Ross, T. K., y Francis, R. A. (1978). The treatment of rusted steel with mimosa tannin. Corrosion Science, 18(4), 351-361. DOI: https://doi.org/10.1016/S0010-938X(78)80049-3

Saji, V. S. (2019). Progress in rust converters. Progress in Organic Coatings, 127, 88-99. DOI: https://doi.org/10.1016/j.porgcoat.2018.11.013

Sancy, M., Gourbeyre, Y., Sutter, E. M. M., y Tribollet, B. (2010). Mechanism of corrosion of cast iron covered by aged corrosion products: Application of electrochemical impedance spectrometry. Corrosion Science, 52(4), 1222-1227. DOI: https://doi.org/10.1016/j.corsci.2009.12.026

Surface, J. Preparation Standard NACE No (Vol. 5). 1/SSPC-SP.

Vetere, V. F., y Romagnoli, R. (1998). Chemical and electrochemical assessment of tannins and aqueous primers containing tannins. Surface Coatings International, 81(8), 385-391. DOI: https://doi.org/10.1007/BF02693869

Xu, W., Han, E. H., y Wang, Z. (2019). Effect of tannic acid on corrosion behavior of carbon steel in $\mathrm{NaCl}$ solution. Journal of Materials Science \& Technology, 35(1), 64-75. DOI: https://doi.org/10.1016/j.jmst.2018.09.001

Zhao, X. D., Cheng, Y. F., Fan, W., Vladimir, C., Volha, V., y Alla, T. (2014). Inhibitive performance of a rust converter on corrosion of mild steel. Journal of Materials Engineering and Performance, 23(11), 4102-4108. DOI: https://doi.org/10.1007/s11665$\underline{014-1199-\mathrm{x}}$

Zhang, X., Xiao, K., Dong, C., Wu, J., Li, X., y Huang, Y. (2011). In situ Raman spectroscopy study of corrosion products on the surface of carbon steel in solution containing $\mathrm{Cl}^{-}$and $\mathrm{SO}_{4}{ }^{2-} . \quad$ Engineering Failure Analysis, 18(8), 1981-1989. DOI: https://doi.org/10.1149/2.013204jes 